\title{
There is more to Context than Location
}

\author{
Albrecht Schmidt, Michael Beigl, and Hans-W. Gellersen \\ Telecooperation Office (TecO), University of Karlsruhe, \\ Vincenz-Priessnitz-Str. 1, 76131 Karlsruhe, Germany \\ albrecht@teco.edu
}

\begin{abstract}
Context is a key issue in interaction between human and computer, describing the surrounding facts that add meaning. In mobile computing research published the parameter location is most often used to approximate context and to implement context-aware applications. We propose that ultra-mobile computing, characterized by devices that are operational and operated while on the move (e.g. PDAs, mobile phones, wearable computers), can significantly benefit from a wider notion of context. To structure the field we introduce a working model for context, discuss mechanisms to acquire context beyond location, and application of context-awareness in ultra-mobile computing. We investigate the utility of sensors for context-awareness and present two prototypical implementations - a light sensitive display and an orientation aware PDA interface. The concept is then extended to a model for sensor fusion to enable more sophisticated context recognition. Based on an implementation of the model an experiment is described and the feasibility of the approach is demonstrated. Further we explore fusion of sensors for acquisition of information on more sophisticated contexts.
\end{abstract}

\section{Keywords}

Adaptive User Interface, Context-Awareness, Handheld Computing, Sensor-based UI, Ultra-Mobile Computing, Wearable Computing,

\section{Introduction}

Context is "that which surrounds, and gives meaning to something else"». Various areas of computer science have been investigating this concept over the last 40 years, to relate information processing and communication to aspects of the situations in which such processing occurs. Most notably, context is a key concept in Natural Language Processing and more generally in Human-Computer Interaction. For instance, state of the art graphical user interfaces use context to adapt menus to contexts such as user preference and dialogue status. A new domain, in which context currently receives growing attention, is mobile computing. While a first wave of mobile computing was based on portable general-purpose computers and primarily focussed on location transparency, a second wave is now based on ultra-mobile devices and an interest in relating these to their surrounding situation of usage. Ultra-mobile devices are a new class of small mobile computer, defined as computing devices that are operational and operated while on the move, and characterized by a shift from general-purpose computing to task-specific support. Ultra-mobile devices comprise for instance Personal Digital Assistants (PDAs), mobile phones, and wearable computers.

A primary concern of context-awareness in mobile computing is awareness of the physical environment surrounding a user and their ultra-mobile device. In recent work, this concern has been addressed by implementation of location-awareness, for instance based on global positioning, or the use of beacons. Location is only one aspect of the physical environment, and as evident from currently reported work location is often used as an approximation of a more complex context. Beyond location, we argue in favor of awareness of further features that contribute to context, in particular awareness of the physical conditions in a given environment, based on the assumption that the more an ultra-mobile device knows about its usage context, the better it can support their user. With advances in sensor technology, awareness of physical conditions can now be embedded in ultra-mobile devices at low cost.

In this paper, we set out with a brief analysis of related work in context-aware mobile computing, followed by introduction of our own working model for context. We will then focus on sensor-based contextawareness and present a prototype that demonstrates the utility of sensor integration in ultra-mobile

${ }^{\dagger}$ Definition of context in The Free On-line Dictionary of Computing 
devices. We will further motivate integration of multiple sensors and sensor fusion to obtain context information that can not be derived from single sensors. We present a model for sensor fusion, describe the development of a context-awareness device, and report on experiments that investigate sensor-based context-awareness.

\section{Related Work}

Context and context-awareness began to be investigated in distributed computing with emergence of mobile computing components in the beginning of the $90 \mathrm{~s}$, led by the desire to support computer usage adequately in varying physical environments. Early investigations were carried out at the Olivetti Research Lab with development of the Active Badge system [17], [18], sensing locality of mobile users to adapt applications to people's whereabouts, and at Xerox PARC with the ubiquitous computing experiment, from which a first general consideration of context-aware mobile computing emerged [14]. Location of use is central to the understanding of context that Schilit et al developed, but their definition of context also includes "the collection of nearby people and objects, as well as changes to those objects over time".

A wide range of systems have been reported, that like Active Badge use only location as context describing their physical environment; these includes systems based on the global positioning system (GPS), and distributed systems using the location information available from underlying communication infrastructure, for instance cell information in cellular networks such as GSM. Leonhardt's work on support of locationawareness contributes generic location service based on a formal model of locality [8]. Only few systems have been described that consider context beyond location. Among these are context-aware information capture and retrieval systems that use time in addition to location, for instance for capture of lecture notes as reported in the Classroom 2000 study 0, and for note-taking in fieldwork as investigated by [10]. A more general concept for time- and location-aware information capture, retrieval and messaging are Brown's Stick-e-notes, documents tagged with location and time information [3], which have been applied in fieldwork and for tour guides [2]. Context-aware information retrieval is also investigated in other tour guide applications, for instance Cyberguide at GeorgiaTech [1] and GUIDE in Lancaster [4]. The latter combines the more obvious contexts of location and user preference with an awareness for quality of service provided by the underlying wireless network

The use of sensors to obtain context information is common in robotics and machine vision applications. With advances in sensor technology, it is now also affordable to embed sensors in ultra-mobile devices, to obtain information about the physical environment, about the handling of ultra-mobile devices and about the user. Relevant developments in this area are the embedding of sensors in handheld computers, the development of sensor devices as independent components, and the integration of sensors in wearable computing systems. In the area of handheld computers, sensor integration is primarily employed for novel interaction techniques. For examples, motion sensors have been integrated with handhelds to extend their interface with awareness of gestures [5], [11]; a recent example is the Rock'n Scroll interface for the Itsy handheld computer [16]. The development of sensor devices as independent components is aimed at more general provision of context-awareness, beyond sensor-enhanced user interfaces. Smith et al have developed a simple context-awareness device integrating a few environmental sensors, as extension for portable and wearable computers [9], [15]. The emergence of wearable computing is further advancing sensor-based context-awareness. Awareness of both the user and the physical environment is considered a distinguishing feature of wearable computers, and a wide range of applications have been prototyped. For instance, biosensors have been used in wearables to for awareness of the user's attention level [6], and the user's emotional statell; simple audio sensing has been employed to obtain an awareness of whether the user is interruptible [13]; and environment sensors have been used to support fieldworkers in mobile workplaces, for awareness of co-located objects and their state [7].

A few key observations can be derived from the analysis of work on context-aware mobile computing. First, the utility of context and context-awareness in mobile computing is evident from reported work, but approaches are hard to relate for lack of a general understanding of what context is. Second, the use of location is dominant but often in approximation of a more general context, for instance guide tours establish the surrounding context indirectly based on location sensing. Third, sensors can be employed to advance context-awareness beyond location-awareness, but obtained context tends to be either of low

\footnotetext{
II www.media.mit.edu/affect/
} 
abstraction (e.g. noise level, temperature, ...) or carefully crafted for specific applications (e.g. user's level of attention).

\section{$3 \quad$ A Working Model for Context-Aware Mobile Computing}

Discussion of context-awareness suffers from the generality of the concept and the lack of models suited for comparison of approaches. In this section we propose a simple working model for context, primarily as means to position our own work on sensor-based context-awareness. The discussion of a context model is followed by consideration of how context can be acquired, and of how it can be applied in ultra-mobile computing.

\subsection{A Working Model for Context}

To structure the concept of context we propose the following model:

- A context describes a situation and the environment a device or user is in.

- A context is identified by a unique name.

- For each context a set of features is relevant.

- For each relevant feature a range of values is determined (implicit or explicit) by the context.

In terms of this model, a hierarchically organized feature space for context can be developed. At the top level we propose to distinguish context related to human factors in the widest sense, and context related to the physical environment. For both general categories we propose further classification into three categories each, as shown in Figure 1. We use the six categories at this level to provide a general structure for context. Within each category, relevant features can be identified, again hierarchically, whose values determine context. Additional context is provided by history, that is by changes in the feature space over time.

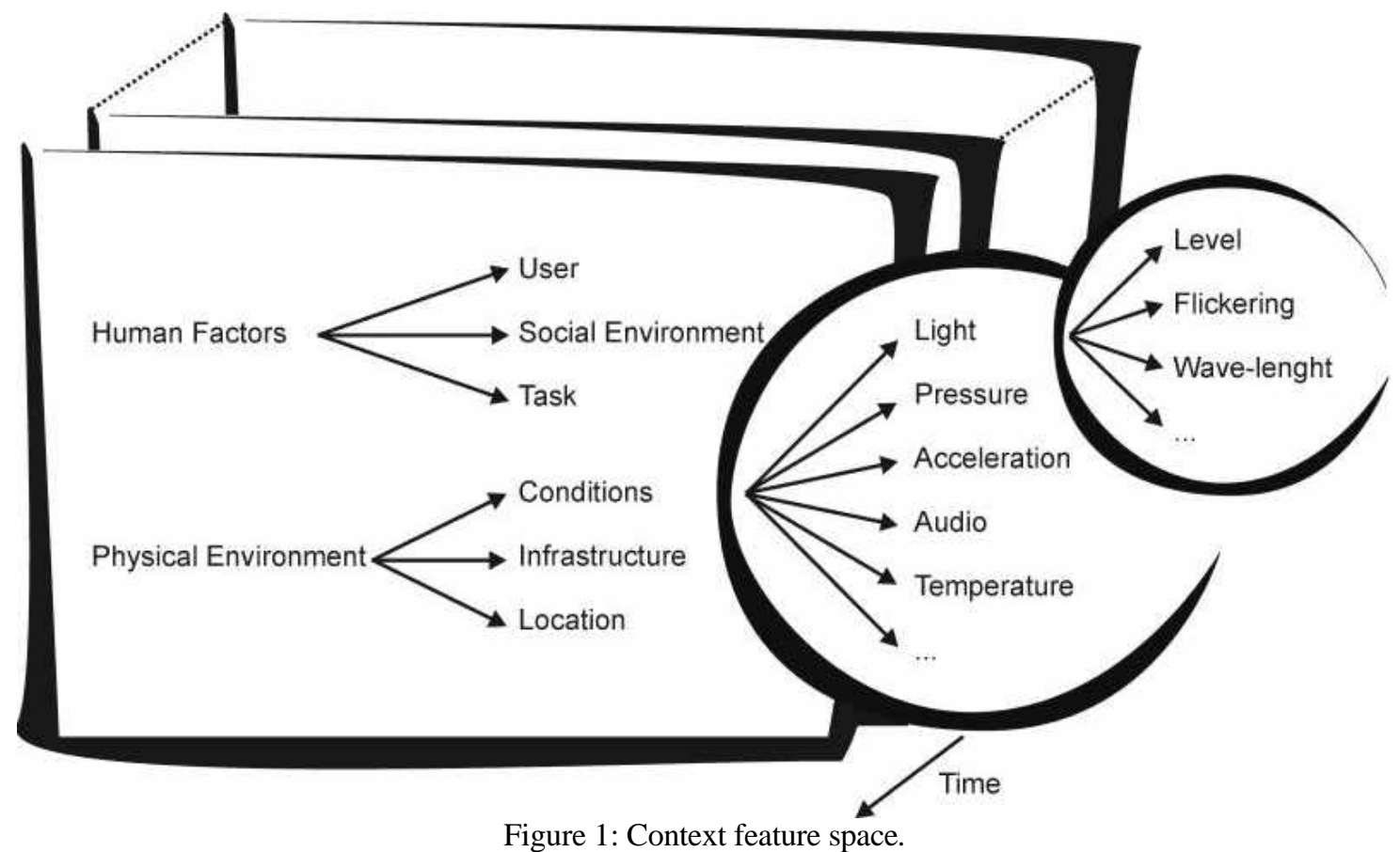

Human factors related context is structured into three categories: information on the user (knowledge of habits, emotional state, biophysiological conditions, ...), the user's social environment (co-location of others, social interaction, group dynamics, ...), and the user's tasks (spontaneous activity, engaged tasks, general goals,...). Likewise, context related to physical environment is structured into three categories: location (absolute position, relative position, co-location,...), infrastructure (surrounding resources for computation, communication, task performance...), and physical conditions (noise, light, pressure,...). 
The described model provides some structure for consideration of context. For pragmatic use of context, the general challenge is to identify the set of relevant features in terms of which a situation or environment can be captured sufficiently. Situations and environments are generally characterized by a large degree of continuity over time, so that context history itself becomes an important feature for approximation of a given situation or environment.

\subsection{Acquiring Context}

In computer applications, context is acquired either explicitly by requiring the user to specify it, or implicitly by monitoring user and computer-based activity. An example for explicit context acquisition in ultra-mobile devices is the specification of current location as required by personal digital assistants (e.g. PSION) to adapt standard location-dependent applications such as clock, tone dialing, and "world". Implicit context acquisition is based on monitoring considitons within the human-computer system; standard examples in ultra-mobile computing are monitoring of user interaction to turn of a device after a period of inactivity, and monitoring of battery power for adaptation of power-intensive applications.

In mobile computing, application usage is set in different environments at different times, constituting changing contexts that lie outside the human-computer-system in the environment. For acquisition of this kind of context there are two approaches. The first is based on smart environments that provide an infrastructure for obtaining context and for providing context to mobile applications. An example is the Active Badge system in which a badge sensing infrastructure that is embedded in the environment, to obtain location information for a range of applications such as message forwarding and teleporting. On a different scale, the GPS system constitutes a smart environment. The second approach is to embed sensors in ultra-mobile devices to acquire context related to the physical environment with the obvious advantage of not having to rely on infrastructure, and to be applicable in any environment. There are examples for sensor-enhanced ultra-mobile device but rather in specialized areas, for instance digital cameras with motion sensors, but generally the use of sensor for context-aware ultra-mobile computing is little developed.

\subsection{Applying Context in Ultra-Mobile Computing}

Independent of particular domains, ultra-mobile computing can benefit from awareness of context in two general ways: first, adaptation to changes in the environment, and secondly improvement of the user interface.

In desktop computing most parameters in the environment are relatively stable, constituting a rather constant context. Likewise, mobile notebook computers are designed for stationary use so that there is usually little variation in the situation surrounding notebook usage. In contrast, the environment of ultramobile computing is characterized by change. Interaction with applications on ultra-mobile computers is usually spontaneous and short; applications are revisited with varying frequency and in different places, without being closed; and ultra-mobile computers can be operational and used while on the move. For instance, a mobile user may use an ultra-mobile computer for Internet telephony while going through a wide range of situations: alone in office, interrupted by a colleague, leaving office, walking to the parking lot, driving off in the car. Ideally the application, in this case telephony, would behave in accordance with the various situations, for instance adapting volume, switching loudspeaker on/off, and so on. This motivates context-awareness to obtain information about usage environments, to be supplied to adaptive applications.

Improvement of human interaction with ultra-mobile devices is a second general motivation for contextawareness. Basically, context information that can be obtained implicitly through awareness, does not have to be obtained explicitly from the user. Context information can be applied to filter the flow of information from application to user, to address the problem of information overload. And for the flow of information from user to application, context information can give additional meaning to the information supplied by the user.

Beyond these general considerations, we have identified three application domains in ultra-mobile computing which we believe can considerably benefit from context-awareness:

- Adaptive user interfaces: the utility of interaction styles and display modes depends largely on the surrounding environment; context-awareness can facilitate adaptation to surrounding conditions. 
- Context-aware communication: universal communication is a driving force for ultra-mobile devices, and techniques are required to filter, reroute, and deliver messages and streams in accordance with a broader communication context, considering issues such as urgency, and interruptibility.

- Proactive application scheduling: context-aware preselection of applications supports the ad hoc style of interaction characteristic for ultra-mobile computing, and their utility for assistance with different tasks in different situations.

After general consideration of context-aware mobile computing in this section, the remainder of the paper will be focussed on sensor-based context awareness. In terms of our working model, the focus is on the category of context relating to physical conditions in mobile computing environments, and on the concept of enhancing ultra-mobile devices with sensors for context-awareness.

\section{$4 \quad$ Enhancing Ultra-Mobile Devices with Sensors}

Advances in sensor technology facilitate integration in ultra-mobile devices. In this section, we will provide a brief overview of available types of sensors, and the context information that can be obtained with them. We present two examples we developed to demonstrate the utility of sensor integration in ultra-mobile devices.

\subsection{Sensor Technology}

Sensor technology is widely applied in robotics, machine vision, and process control. Advances with respect to issues such as size, power consumption, processing requirements, and cost-effective production enable their integration in ultra-mobile devices. As ultra-mobile computing products tend to compete primarily over price, cost of add-on technology is very critical, and hence we focus on simple and affordable technologies in this overview.

Optical/Vision. Single optical sensors (e.g. photo-diode, color sensor, IR and UV-sensor, etc.) supply information on the light intensity, the density, the reflection, the color temperature of the light (wavelength), and the type of light (sunlight, type of artificial light, etc). A camera, seen as an array of light sensors, can supply visual information of the environment from which simple cues can be obtained with little processing (e.g. main color, motion); at higher processing cost richer context can be extracted (e.g. detection of objects, landmarks, people, gestures etc).

Audio. A wide range of methods at different processing cost is available to extract context information from audio data to analyze the data is available. With little effort (i.e. cheap microphone and some electronics) loudness, type of background noise, and base frequency can be determined. With more advanced audio sensing and processing, e.g. for speaker identification, more sophisticated context can be obtained. An interesting aspect is also use of ultrasonic sensors, for instance to augment human sensory capabilities.

Motion. Ultra-mobile devices are used while on the move, and sensing of the inclination, motion or acceleration of the device is of obvious interest. Typical sensors are mercury switches, angular sensors, and accelerometers. Contexts like moving the device, having the device stationary on a table, and driving in a car can be indicated by the data provided by an accelerometer.

Location. Position, location, co-location, and proximity of users, devices, and environment provide important context in ultra-mobile computing. Outdoors, GPS is often used for fine grained location sensing but coarse location information is also available from cellular network infrastructures such as GSM. Indoors, location sensors are typically embedded in the environment, as for instance in the Active Badge system. Co-location can be sensed for instance using radio beacons.

Bio-Sensors. Ultra-mobile computers are typically personal, and awareness of their user can be improved by use of biosensors that for instance measure as pulse, skin resistance, and blood pressure, to obtain context useful in sports and medical applications; with added processing, an awareness of e.g. emotional state of the user may be obtained.

Specialized sensors. Sensors for touch, temperature, air pressure, and so on, may be integrated for use in more specialized applications. For particular mobile work settings, further sensors may be integrate in ultra-mobile deviecs, for instance sensor measuring gas concentration and radiation, to augment the sensory capabilities of their users, and to facilitate automated information capture. 


\subsection{Sensor-based Context-Awareness for Adaptive PDA User Interfaces}

In section 3.3 we pointed out that adaptive user interfaces are an important application of contextawareness in ultra-mobile computing. Below, we describe two prototypes of adaptive PDA user interfaces, that we build based on integration of low-cost sensor technology.

\section{Light-Sensitive Display}

Integration of simple sensors can substantially add to usability of ultra-mobile devices. In a straightforward demonstrator we integrated a light sensor in a Palm Pilot, to provide it with an awareness of surrounding lighting conditions, which can be applied for control of its display's backlight. This use of light sensors is common in our everyday environments, and used in many consumer products. Surprisingly, it has not yet been used in mobile and ultra-mobile computer products, probably due to the fact that product concept definitions are still primarily based on conventional computer product concepts developed for stationary environments, which is also evident in the persistence of direct manipulation interaction styles, and desktop metaphor.
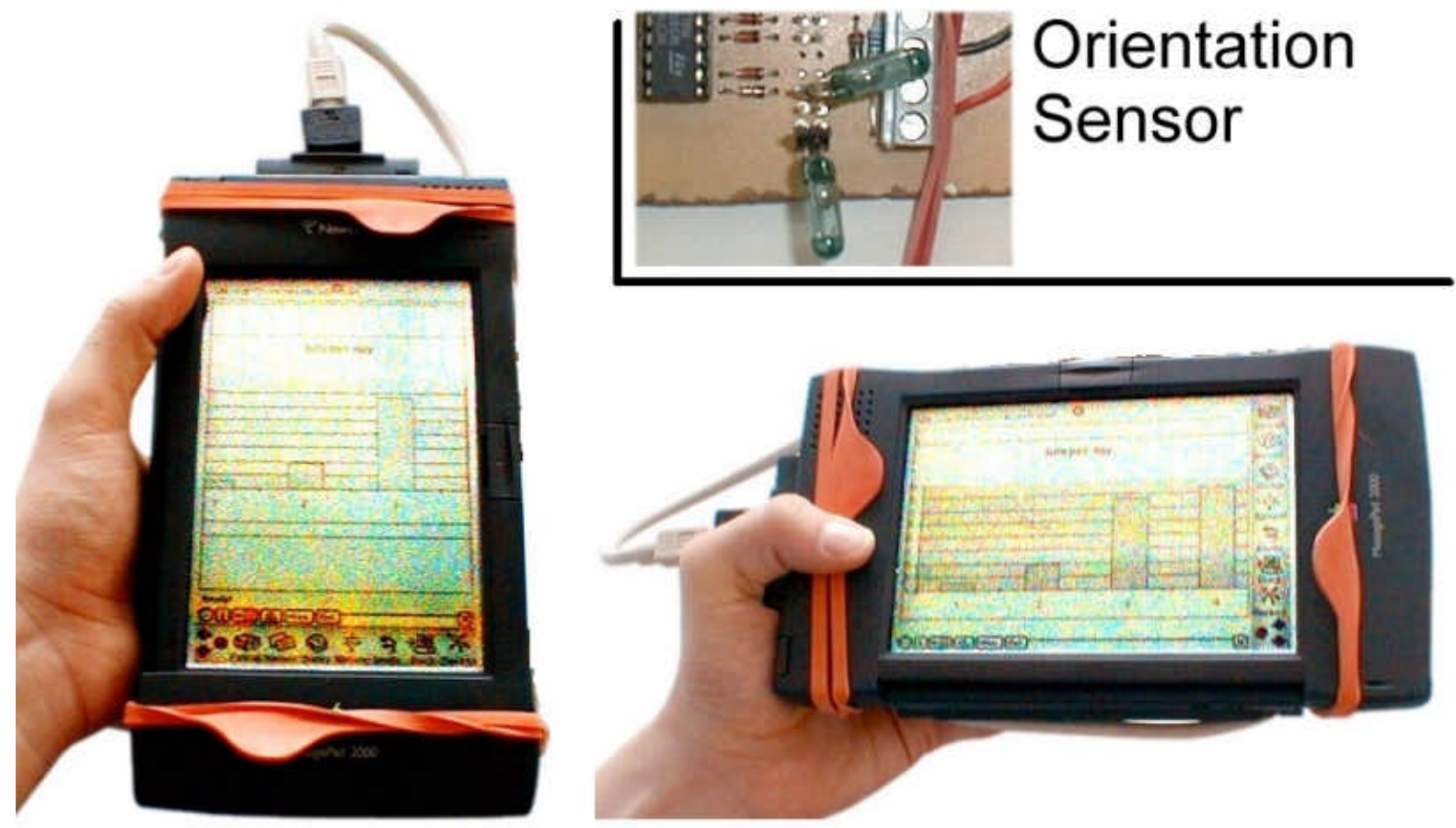

Figure 2: Using the orientation-aware device in different modes.

\section{Orientation-Sensitive User Interface}

As a more elaborate demonstrator for sensor-based context-awareness in ultra-mobile computers we have enhanced a PDA, in this case a Newton MessagePad, with awareness for its orientation by adding two mercury switches and simple electronics. In this demonstrator, the orientation of the user interface is adapted to the orientation of the device: if the device is held upright the user interface is displayed in the usual portrait mode, if it is turned sideways the user interface is switched to landscape mode, and if it is further turned upside down the user interface is also rotated accordingly. The Newton MessagePad and also other PDAs actually offer operating system support for rotation of the user interface but experience shows that this feature is hardly used, if at all. This is not surprising as it does not make sense to define user interface rotation a priori and out of context; in conjunction with awareness of device orientation though user interface rotation can be used very effectively for improvement of human-computer interaction: 
- Always right way up: the adaptive user interface is always the right way up whichever way a device is held; when a device is picked up, it is the system that adapts user interface orientation, instead of the user adapting device orientation.

- Alternating between Portrait and Landscape: The user can change from portrait to landscape view of documents by simply turning the device; this can be very useful for viewing data organized in two dimension, for instance spreadsheets; the option of resorting to landscape view is also useful for browsing documents that otherwise would require vertical scrolling.

- Collaboration over PDAs: in face-to-face settings, users can take turns in interacting with the same PDA by simple handing it over with out having to turn it; a user can show the display to another by simply tilting it toward them.

\section{$5 \quad$ Sensor Fusion for Context Awareness}

In the previous section we have reported work demonstrating the utility of enhancing ultra-mobile devices with simple and affordable sensor technology. In these examples, context information obtained from sensors is at a low level of abstraction, capturing isolated features of a situation: "dark vs. bright" and "device orientation". In this section, we present a new sensor-based approach for obtaining context information at a level of abstraction that relates more to situations than to specific physical conditions, for instance "indoors/outdoors", "engaged in conversation", "in a meeting", and so on. Our approach is based on fusion of multiple simple sensors in an awareness component, and on association of patterns in the sensory data with specific context. We describe an experiment, based on statistical analysis of sensor data and specification of rules for context recognition. The work reported in this section is conducted within the ESPRIT Project TEA "Technology for Enabling Awareness". General objectives of this project are to assess which sensors can be combined effectively for context-awareness, which methods are required to relate sensory data to situations, and how complex contexts can be constructed from simpler ones.

\subsection{Architecture for Sensor Fusion}

We developed a layered architecture for multi-sensory context-awareness, with sensor, cue, context, and scripting layers that provide successive abstraction from physical conditions measured in a given situation.

Sensors. At this level, we distinguish physical and logical sensors. Physical sensors are electronic hardware components that measure physical parameters in the environment. All information gathered from the host of the awareness component (i.e. the sensor-enhanced ultra-mobile device) are considered as logical sensors. Each sensor is regarded as a time dependent function that returns a scalar, a vector, or a symbolic value. A set (finite or infinite) of possible values for each sensor is defined.

Cues. The concept of Cues provides an abstraction from physical and logical sensors. For physical sensors introducing of cues as abstraction also solves the calibration problem. A cue is regarded as a function taking the values of a single sensor up to a certain time as input and providing a symbolic or sub-symbolic output. A set (finite or infinite) of possible values for each cue is defined. Each cue is dependent on a single sensor but different cues may be based on the same sensors.

Contexts. A context is a description of the current situation on an abstract level, derived from the cues that are available. The context is described by a set of two-dimensional vectors. Each vector consists of a symbolic value describing the situations and a number indicating the certainty that the user (or the device) is currently in this situation. The finite set of the symbolic values is defined.

Scripting. This layer provides mechanisms to include context information in application. Supported semantics are entering a context, leaving a context, and while in a context.

- Entering a context: if a certain situation is indicated with a probability that is higher than a threshold an action is performed after a certain time".

- Leaving a context: if the probability for a certain situation is becoming less than a threshold an action is performed after a certain time.

- While in a context: if a certain situation is indicated with a probability higher than a threshold an action is performed every specified time interval.

\footnotetext{
\# In case of context prediction the time might be negative.
} 


\subsection{An Awareness Device for Experimentation}

For experimentation with multi-sensory context-awareness, we built an Awareness Device with both hardand software components. Basic signal processing is done in hardware, while cue generation, calculation of contexts, and script execution is done in software. The implementation that we describe is a first prototype design, focussed on data acquisition, rather than elegant processing. The first hardware platform simply samples sensor data and packages it into a digital signal that can be transferred to a standard portable computer for further processing. The data collection unit was designed to allow experimentation with a greater number of possibly relevant sensors, although it is anticipated that some of the used sensors may not be relevant for awareness of investigated situations.

The data traverses the system through four major blocks: the sensors, the analog-to-digital converter, the microcontroller, and the serial line. The sensors measure conditions in the environment and translate them to analog voltage signals on a fixed scale. These analog signals are converted to digital signals and passed to the microcontroller. The microcontroller oversees the timing of the analog-to-digital converter and the sensors, and converts the data from the analog-to-digital converter's bus to the serial line. Finally, the serial line connects to a host computer for further processing, see Fig. 3.

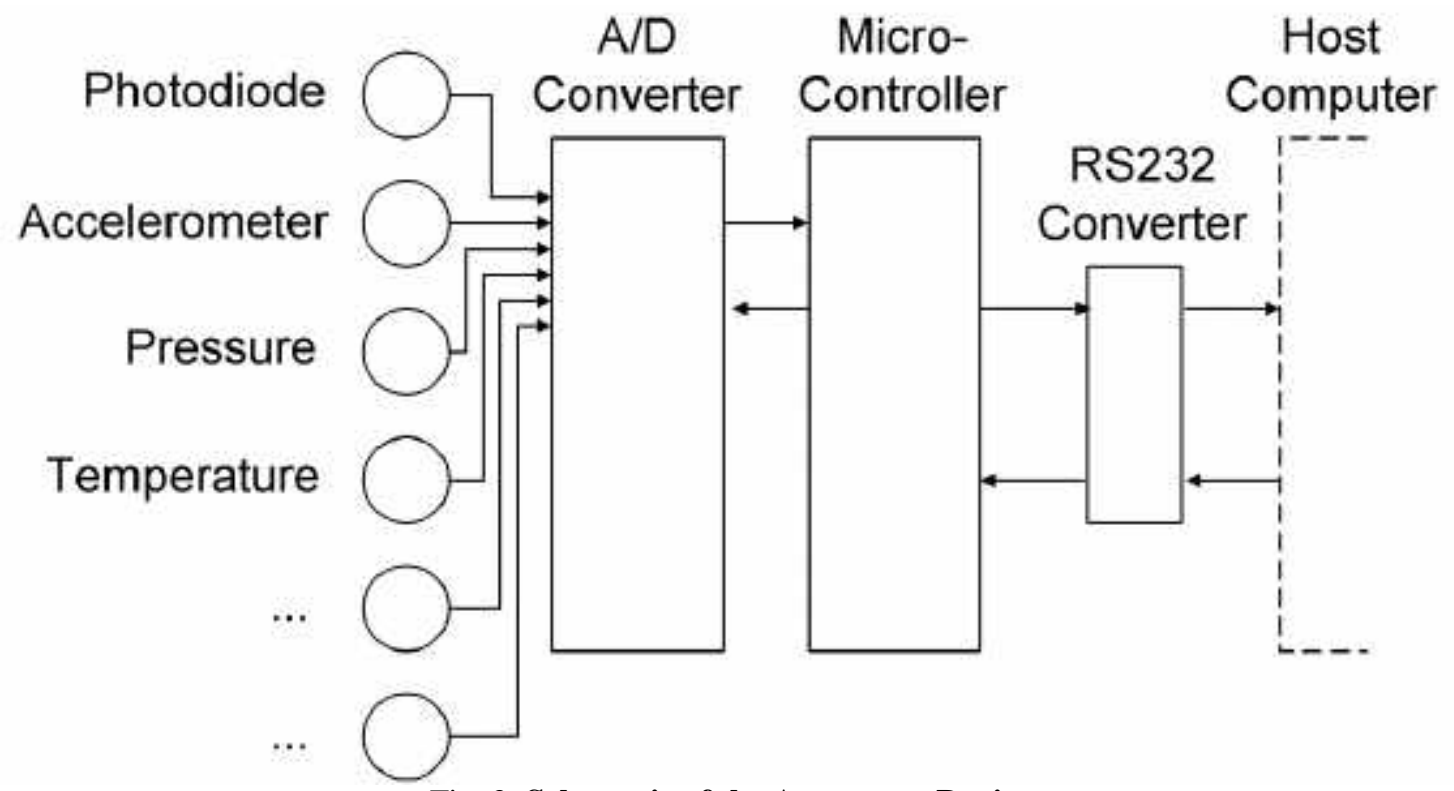

Fig. 3: Schematic of the Awareness Device

\subsection{Deriving Context from Sensor Data}

The awareness device provides about 1100 Bytes of sensor readings per second. The data from different sensors is transmitted interlaced to ensure recognition of sudden changes in the readings. To derive context information, sensor data is processed according to the architecture described above with a two-step mapping from sensor to cues and from cues to context. For experimentation with this approach, we collected raw data in different situations, for instance "indoors" and "outdoors". From the interlaced stream of sensory data, vectors are extracted that represent individual sensor readings over a time interval.

As an example for obtained sensor readings, Figures 4 shows data obtained from a simple light sensor in two different contexts. Obviously, situations with different lighting conditions as in this example, can be easily and reliably distinguished. Simple statistical functions can be applied to extract cues such as "artificial light" and "brightness" from the light sensor readings. Similarly, other sensor readings can be abstracted to cues, for instance accelerometer data to "stationary", "walking", and so on. These examples show that a larger number of cues can be obtained from simple sensors. 


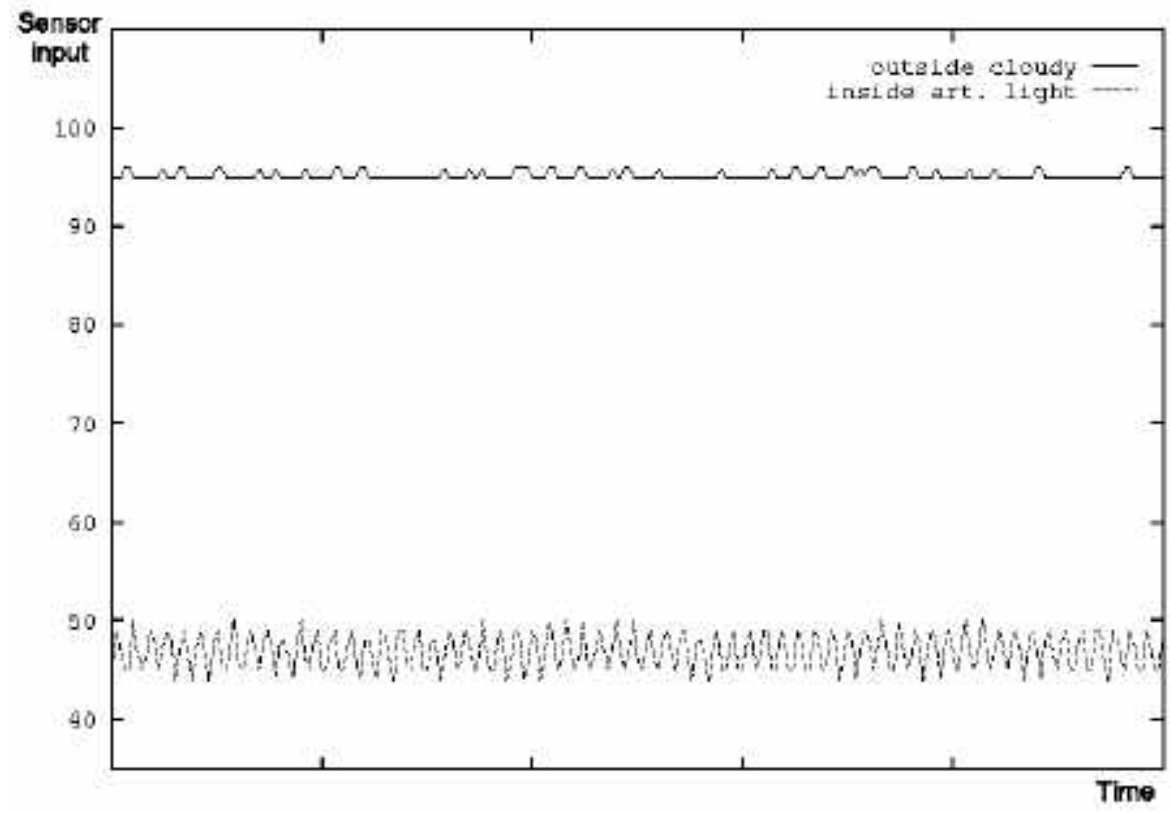

Fig. 4: Light sensor data

The cues viewed independently may not be applicable for context-awareness but our assumption is that many situations can be sufficiently described by a set of cues. To illustrate this concept, table 1 lists some cues that can contribute to description of specific contexts. For the cue to context mapping we foresee to approaches. The first is explicit rule specification to infer context from cues, informed by prior statistical analysis. The second is to use artificial intelligence methods to relate cues to specific contexts in a learning process.

\begin{tabular}{l|l} 
Context & Cues \\
\hline In the office & Artificial light, stationary or walking, room temperature, dry \\
\hline Jogging & Natural light (cloudy or sunny), walking or running, dry or raining, high pulse
\end{tabular}

Tab. 1: Describing Contexts in Terms of Cues

First experiments with the described approach showed that is useful to introduce the abstraction of cues over raw sensor data. Having these abstractions it becomes much easier to explicitly describe contexts in terms of conditions that define or debar a context. Also when deploying automatic mappings, such as artificial neural networks, the introduced abstraction reduces the input dimension and eases the construction process.

\section{Conclusion}

In this paper we showed that context-awareness can enhance ultra-mobile devices, especially towards providing an improved interface between the user and the device. To explore context we introduced a working model and discussed basic application domains. To gain context information the usage of sensors is proposed. Here we gave an overview of available technology that can be used for acquiring information from the user and the environment. Then we presented two prototypical implementations of ultra-mobile devices that were enhanced using simple sensors, namely a light sensitive display and an orientation aware PDA, that proofed the concept.

Furthermore we introduced an approach for context-awareness based on fusion of multiple sensors. The architecture consists of sensor, cue, context and scripting layer. An awareness device based on this architecture was implemented and presented together with an example for deriving contexts from multiple sensors. First experiments also showed that deploying multiple sensors of the same type (e.g. several light sensors) contributes valuable additional information (e.g. direction of light, sunny, shade, etc.).

Our current research focus is on the automation of the sensor fusion process. We investigate different approached for feature extraction and dimension reduction to optimize sensor to cue mapping. We compare 
different AI-techniques and neural networks to find a most suitable solution for automated context detection. To provide a simple inclusion of context information in applications we work on a context description protocol based on XML and on programming primitives ease programming.

A further research topic we are working on is cooperative context discovery. Here information acquired by networked sensor enhanced ultra-mobile devices as well as by a smart environment is used to establish context information.

\section{$7 \quad$ References}

Abowd, G.D., Atkeson, C.G, Brotherton, J.A., Enqvist, T., Gully, P., Lemon, J. Investigating the capture, integration and access problem of ubiquitous computing in an educational setting. In the Proceedings of CHI '98, May, (1998).

[1] Abowd, G.D., Atkeson, C.G., Hong, J., Long, S., Kooper, R., Pinkerton, M. Cyberguide: A Mobile Context-Aware Tour Guide. ACM Wireless Networks 3. 421-433. (1997).

[2] Brown, P. J., Bovey, J. D., Chen, X. Context-Aware Applications: From the Laboratory to the Marketplace. IEEE Personal Communications, 4(5) pp.58-64. (1997).

[3] Brown, P.J. The Stick-e Document: a Framework for Creating Context-Aware Applications. Electronic Publishing '96 259-272 (1996).

[4] Cheverst, K., Mitchell, K., Davies, N. (1998). Design of an Object Model for a Context Sensitive Tourist GUIDE. Proceedings of the International Workshop on Interactive Applications of Mobile Computing (IMC98), Rostock, Germany, November (1998).

[5] Harrison, B.L., Fishkin, K.P., Gujar A., Mochon, C., Want, R. Squeeze Me, Hold Me, Tilt Me! An Exploration of Manipulative User Interfaces. In the Proceedings of CHI '98, May, (1998).

[6] Healey, J. and Picard, R.W. StartleCam: A Cybernetic Wearable Camera. Proceedings of the Second International Symposium on Wearable Computing, Pittsburgh, PA, October 19-20, (1998).

[7] Korteum, G., Segall, Z., Bauer, M. Context-Aware, Adaptive Wearable Computers as Remote Interfaces to "Intelligent" Environments. . Proceedings of the Second International Symposium on Wearable Computing Pittsburgh, PA, October 19-20, (1998).

[8] Leonhard, U., Magee, J., Dias, P. Location Service in Mobile Computing Environments. Computer \& Graphics. Special Issue on Mobile Computing. Volume 20, Numer 5, September/October (1996).

[9] Maguire, G. Q. Jr., Smith, M. T, and Beadle, H. W. P. "SmartBadges: a wearable computer and communication system" Codes/CASHE '98, 16-March 1998, Seattle, Washington, U.S.A. (1998).

[10] Pascoe, J. Adding Generic Contextual Capabilities to Wearable Computers. Proceedings of the Second International Symposium on Wearable Computing, Pittsburgh, PA, October 19-20, (1998) [11] Rekimoto, J. Tilting operations for small screen interfaces. UIST '96. Proceedings of the ACM symposium on User interface software and technology, pages 167-168, (1996).

[12] Ryan, N., Pascoe, J., Morse, D. Enhanced Reality Fieldwork: the Context-Aware Archaeological Assistant. In: Computer Applications in Archaeology Gaffney,V., van Leusen, M., Exxon, S. (eds.).(1997)

[13] Sawhney, N., and S., Chris. "Nomadic Radio: A Spatialized Audio Environment for Wearable Computing." Proceedings of the International Symposium on Wearable Computing, Cambridge, MA, October 13-14, (1997).

[14] Schilit, B.N., Adams, N.L., Want, R. Context-Aware Computing Applications. Proceedings of the Workshop on Mobile Computing Systems and Applications, Santa Cruz, CA, December. IEEE Computer Society (1994).

[15] Smith, M.T., Sensors in Wearables. International Conference on Wearable Computing. Stuttgart, Germany. (1999).

[16] Waldspurger, C., The ITSY Pocket Computer. Invited talk Proceedings of the Second International Symposium on Wearable Computing, Pittsburgh, PA, October 19-20, http://www.research.digital.com/wrl/itsy/talk-iswc98/. (1998).

[17] Want, R., Hopper, A., Falcao, V., Gibbons, J. The Active Badge Location System. ACM Transactions on Information Systems 10(1) pp 91-102, (1992).

[18] Ward, A., Jones, A., Hopper, A. A New Location Technique for the Active Office. IEEE Personal Communications 4(5) 42-47, (1997). 